\title{
M51 ULX-7: super-orbital periodicity and constraints on the neutron star magnetic field
}

\author{
G. Vasilopoulos ${ }^{1 \star}$, S. K. Lander ${ }^{2}$, F. Koliopanos ${ }^{3,4}$, C. D. Bailyn ${ }^{1}$ \\ ${ }^{1}$ Department of Astronomy, Yale University, PO Box 208101, New Haven, CT 06520-8101, USA \\ ${ }^{2}$ Nicolaus Copernicus Astronomical Centre, Polish Academy of Sciences, Bartycka 18, PL-00-716 Warsaw, Poland \\ ${ }^{3}$ CNRS, IRAP, 9 Av. colonel Roche, BP 44346, F-31028 Toulouse cedex 4, France \\ ${ }^{4}$ Université de Toulouse; UPS-OMP; IRAP, Toulouse, France
}

Accepted XXX. Received YYY; in original form ZZZ

\begin{abstract}
In the current work we explore the applicability of standard theoretical models of accretion to the observed properties of M51 ULX-7. The spin-up rate and observed X-ray luminosity are evidence of a neutron star with a surface magnetic field of $2-7 \times 10^{13} \mathrm{G}$, rotating near equilibrium. Analysis of the X-ray light-curve of the system (Swift/XRT data) reveals the presence of a $\sim 39 \mathrm{~d}$ super-orbital period. We argue that the super-orbital periodicity is due to disc precession, and that material is accreted onto the neutron star at a constant rate throughout it. Moreover, by attributing this modulation to the free precession of the neutron star we estimate a surface magnetic field strength of $3-4 \times 10^{13} \mathrm{G}$. The agreement of these two independent estimates provide strong constraints on the surface polar magnetic field strength of the NS.
\end{abstract}

Key words: X-rays: binaries - galaxies: individual: M51 - stars: neutron - pulsars: individual: M51 ULX-7

\section{INTRODUCTION}

The discovery of pulsating ultra-luminous X-ray sources (PULXs) demonstrated that neutron stars (NSs) can sustain accretion at a super Eddington rate, assuming observed fluxes translate to isotropic luminosities (Bachetti et al. 2014). This discovery has fueled a search that led to the discovery and study of more PULXs in recent years (e.g. Carpano et al. 2018; Israel et al. 2017; Bachetti et al. 2014; Fürst et al. 2016; Rodríguez Castillo et al. 2019; Sathyaprakash et al. 2019). Furthermore, based on spectral similarities between pulsating and non-pulsating ULXs, there is now compelling evidence in favor of highly magnetized NSs being the engines of ULXs (Koliopanos et al. 2017; Walton et al. 2018a).

In order to explain the super-Eddington luminosities of PULXs, it has been speculated (Mushtukov et al. 2015) that the NSs in PULXs must have higher magnetic fields strengths $\left(B>10^{13} \mathrm{G}\right)$, than NSs in typical X-ray pulsars (Ho et al. 2014; Christodoulou et al. 2016). However, the radiative mechanisms of the NS accretion column (Becker \& Wolff 2007) have not yet been fully studied at super-Eddington accretion rates, where several assumptions break due to the accretion column geometry (West et al. 2017). Nevertheless, in some PULXs (see NGC300 ULX1) steady accretion at super Eddington rates and a NS with typical magnetic field (dipole term; 10 $12 \mathrm{G}$ ) can coexist (Walton et al. 2018b; Vasilopoulos et al. 2018, 2019).

^ E-mail: georgios.vasilopoulos@yale.edu
A mechanism that could affect the long term behaviour of PULXs is the transition from accretor to propeller regime as the accretion disc inner radius changes size and becomes larger than the NS corotation radius (Illarionov \& Sunyaev 1975). This transition is often seen during the decay of outbursts from transient X-ray pulsars (Tsygankov et al. 2016b) like Be X-ray binaries (BeXRBs). Among PULXs the best candidate system for observing such transition is M82 X-2, a system that has shown a bimodal distribution in its X-ray flux (Tsygankov et al. 2016a), while the NS exhibits episodes of both spin-up (short term) and spin-down (secular evolution) that are consistent with propeller transition (Bachetti et al. 2019).

Another important characteristic of ULXs is the presence of ultra-fast outflows (UFO) (e.g. Pinto et al. 2016; Kosec et al. $2018 \mathrm{~b}, \mathrm{a})$. These outflows originate from the disc as radiation pressure becomes important (Poutanen et al. 2007). Moreover, outflows should be optically thick (Abolmasov et al. 2009), and thus allow radiation to escape from a hollow funnel, resulting in mild to moderate beaming (King et al. 2017; King \& Lasota 2019). The radiation originating from the central source can be scattered through the funnel walls, thus dramatically changing the temporal and spectral signatures of the signal seen by an observer that views the funnel under different orientations (Abolmasov et al. 2009; Middleton et al. 2015). In the case of magnetized NSs as central engines, a large fraction of the pulsed emission can be scattered though the funnel walls, resulting in a decrease of the pulsed fraction (King et al. 2017). Interestingly, models that account for changes of the 
geometry of the system (e.g. due to precession: Middleton et al. 2018 b , 2019) have been proposed to explain the super-orbital modulations of the X-ray light-curves of ULX systems (Dauser et al. 2017; Middleton et al. 2018a).

However, the driving engine behind the disc and outflow precession is still unclear, and could be a combination of different physical processes. There are numerous torques that act on the accretion disc and can cause its distortion (i.e. warping) and change in orientation (tilt of disc) (Shakura et al. 1998, 1999). These are: the tidal force from the companion massive star, the interaction with the magnetosphere of the NS, or the irradiation of the disc (Pringle 1996; Foulkes et al. 2010; Ogilvie \& Dubus 2001). One can argue that the presence of super-orbital periodicity in the X-ray lightcurves of ULX is not necessarily evidence of outflows. After all many X-ray binaries, like Her X-1, show such behaviour on similar timescales.

The basic observable quantities of PULXs are the flux and super-orbital variability often seen in X-rays, the NS spin as well as the spin-up/down rate. These observables should provide the basis for testing accretion theory in extreme accretion rates, and compare with predictions of classical (i.e. not super-Eddington) models. A major question is how does the accretion disc behave given the high accretion rates, and how do the disc properties alter the torque acting onto the NS and the variations of the observed X-ray flux. In a recent study, Chashkina et al. (2019) provide theoretical semianalytical calculations for the inner disc radius and mass accretion onto the NS during super-Eddington accretion, by taking into account advection of heat and mass loss due to a wind originating from the disc (see also, Mushtukov et al. 2019).

To put things into perspective, we are now at a stage where we can test standard accretion theory in PULXs, and compare with numerical solutions that take into account more detailed physical properties. But most importantly, by obtaining deep X-ray observations, and by performing long X-ray monitoring campaigns we can investigate different aspects of ULXs and obtain self-consistent solutions for their properties. We can then answer questions like: do PULXs host NSs with unusually high magnetic fields? are observed X-ray fluxes enhanced by beaming or are a product of super-Eddington mass-accretion rate? are outflows a necessary requirement for super-orbital modulation in the X-ray light-curves of PULXs?

A particularly interesting system is M51 ULX-7, a known ULX system located in the Whirlpool galaxy at a distance of (8.58 \pm 0.1$)$ Mpc (McQuinn et al. 2016). The detection of coherent pulsations $(P \sim 2.8 \mathrm{~s})$ and an orbital period of $\sim 2 \mathrm{~d}$ confirm the system as a new PULX (Rodríguez Castillo et al. 2019). The X-ray spectral and temporal properties of the system have been presented by Rodríguez Castillo et al. (2019).

In regard to the spin-evolution of M51 ULX-7, temporal analysis of XMM-Newton data enabled the measurement of the NS spin in 4 epochs (Rodríguez Castillo et al. 2019). According to the authors, between MJD 53552 and MJD 55723 the spin period of the NS evolved from 3.2831(2) s to 2.8014(7) s, yielding a secular spinup of $\dot{P}=-2.57 \times 10^{-9} \mathrm{~s} / \mathrm{s}$. Between MJD 55723 and MJD 58284 the spin period of the NS evolved from 2.8014(7) to 2.7977148(2) s, yielding a secular spin-up of $\dot{P}=-1.67 \times 10^{-11} \mathrm{~s} / \mathrm{s}$. These intervals represent two epochs with different secular spin evolution, one being significantly slower than the other. The intrinsic spin-up of the system could only be constrained from the combination of two XMM-Newton observations (span of 2 days), and was determined to be $\dot{P}=-2.4 \times 10^{-10} \mathrm{~s} / \mathrm{s}$ (or $\dot{v}=3.1 \times 10^{-11} \mathrm{~Hz} / \mathrm{s}$ ) on MJD 58283.44. Finally, based on the orbital solution obtained for the system, a lower limit on the mass of the companion has been placed at $8.3 M_{\odot}$ (Rodríguez Castillo et al. 2019).

In regard to the spectral properties of M51 ULX-7, Rodríguez Castillo et al. (2019) have performed spectral analysis on archival $X M M$-Newton observations of the system. The system was detected during a hard high flux state and a soft low flux state. For the observations obtained in the hard state, the spectra were similar and can be fitted by a dual thermal component (see application to ULXs; Koliopanos et al. 2017; Walton et al. 2018a). The soft component has a temperature of $\sim 0.4-0.5 \mathrm{keV}$ and a size of $700-800 \mathrm{~km}$ while the hard component has a temperature of $1.33-1.5 \mathrm{keV}$ and a size of $90-100 \mathrm{~km}$. The fit to the X-ray spectra yields an unabsorbed X-ray luminosity $\left(L_{\mathrm{X}}\right)$ of $5.6-7.1 \times 10^{39} \mathrm{erg} \mathrm{s}^{-1}(0.3-10.0 \mathrm{keV})$. For one observation (obs L; where L refer to low flux state) the spectrum was greatly different from the remaining observations and could be fitted by a single-temperature ( $\mathrm{keV} \sim 0.19 \mathrm{keV})$ black body component with a size of $\sim 1700 \mathrm{~km}$. The absorption corrected flux during this observation was estimated to be $3 \times 10^{38} \mathrm{erg} \mathrm{s}^{-1}(0.3-10.0 \mathrm{keV})$. However, we note that Rodríguez Castillo et al. (2019) did not account for the possibility that the decrease in the observed flux is due to occultation. In general, it has been shown that the observed flux of ULXs can significantly underestimate the intrinsic X-ray luminosity if one does not account for the obscuration by outflows and/or a precessing disc (e.g. Vasilopoulos et al. 2019; Carpano et al. 2018; Fabrika et al. 2015).

In this work we will focus on the study of M51 ULX-7. In section $\$ 2$, we will present the X-ray light-curve of the system and discuss the presence of steady super-orbital periodicity. In section $\S 3$ we will discuss the basic predictions of standard accretion theory onto a magnetized NS, and use them to put constraints onto the magnetic field strength of the NS in a self-consistent manner. Moreover, we will investigate whether our findings change by accounting for changes in the accretion disc structure due to the high accretion rate. We will do so by invoking the semi-analytical calculations of Chashkina et al. (2019). We will show that M51 ULX-7 cannot be in the so-called slow rotator regime, and the most probable solution is that the NS accretes at an almost constant accretion rate and is near spin equilibrium. In section 4 we will discuss the nature of the super orbital modulation, and argue that outflows are not a necessary condition for causing the observed pattern of variability, and a precessing disc could be a valid mechanism. A compelling engine that could drive the disc precession is free precession of the system's NS. This then leads to an estimate for how distorted the star is, and thus - accounting for the superconducting state of the NS core - enables us to estimate the NS magnetic field required to provide this distortion.

\section{DISCOVERY OF SUPER-ORBITAL PERIODICITY}

X-ray observations of M51 have been obtained by the Neil Gehrels Swift Observatory (Swift, Gehrels et al. 2004) X-ray Telescope (XRT, Burrows et al. 2005). To investigate the long-term variability of the system we searched for available Swift/XRT observations in the direction of M51. We found about 150 Swift/XRT observations, where $\sim 70$ were performed between MJD 55715-55795 (Epoch I) and the remaining cover a span of about 500 days between MJD 58215-58715 (Epoch II). We note that both epochs I and II correspond to the period of slow spin-up of M51 ULX-7 as defined in the previous section (i.e. MJD 55723-58282). Data were 

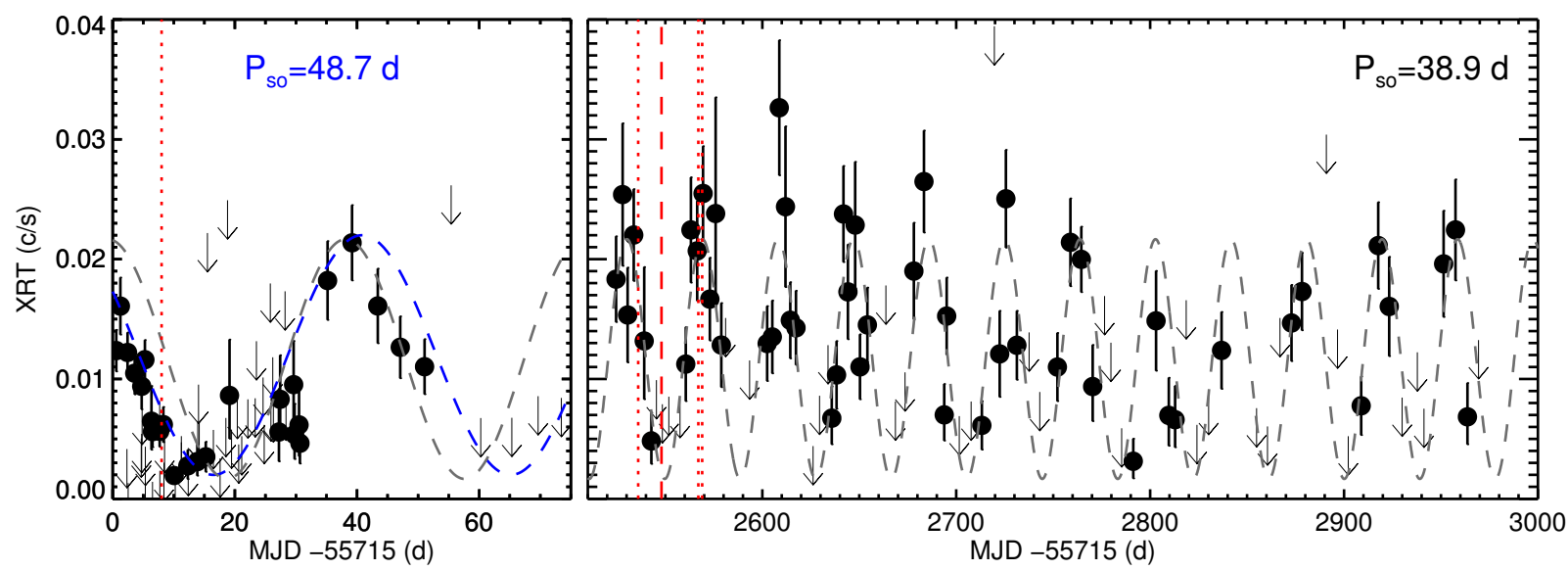

Figure 1. X-ray light curve of M51 ULX-7 based on Swift/XRT monitoring of the region for two different epochs. For non-detections, arrows represent $3 \sigma$ upper limits. Dashed gray sinusoidal curve is plotted to guide the eye, its $38.97 \mathrm{~d}$ period based on the periodicity detected during the second epoch (right plot). The curve is extrapolated to the earlier epoch for illustration purposes; we see that the early data points fit the curve well, hinting that the periodicity could have remained in phase for the intervening $\sim 60$ cycles for which we have no data. However, given the putative periodicity $\sim 48.7 \mathrm{~d}$ we find for the first epoch (see Fig. 2) and the inconsistency of the gray curve with the non-detections after MJD55715 = $60 \mathrm{~d}$, we also present a second curve (blue line) in better agreement with these findings. Vertical red lines mark the epoch where XMM-Newton observations have been performed (Rodríguez Castillo et al. 2019). A super-soft spectrum has been detected during the observation marked with dashed red line (see text for details). For all XMM-Newton observations marked with dotted red lines a NS spin period has been detected (Rodríguez Castillo et al. 2019).

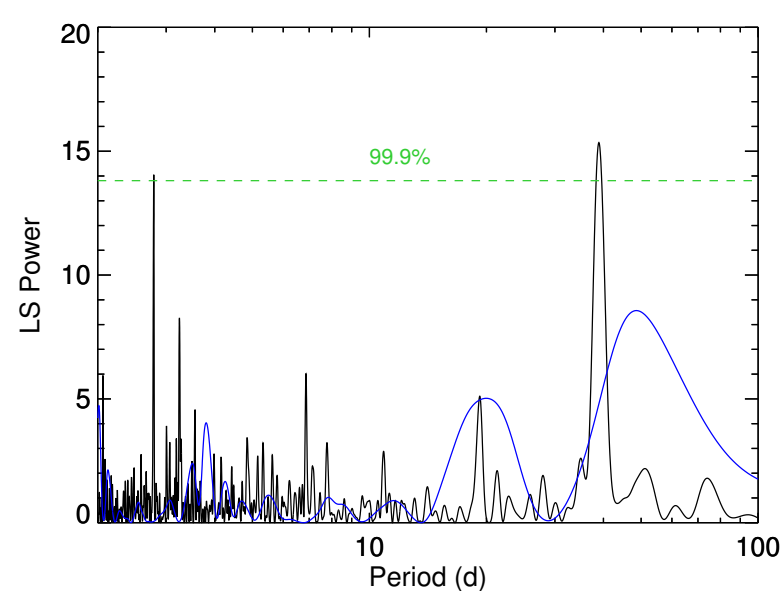

Figure 2. Lomb-Scargle periodogram for epoch I (blue line) and epoch II (black line) as defined in Fig. 1. Horizontal dotted line marks the $99.9 \%$ confidence level based analysis of simulated light-curves.

retrieved and analyzed though the UK Swift science data centre ${ }^{1}$, using standard procedures as outlined in Evans et al. (2007, 2009). To further investigate spectral changes we estimated the spectral Hardness Ratio (HR) from each observation. HR is defined as the ratio of the difference over the sum of the number of counts in two subsequent energy bands: $H R=\left(R_{i+1}-R_{i}\right) /\left(R_{i+1}+R_{i}\right)$, where $R_{i}$ is the background-subtracted count rate in a specific energy band. For this study we used the $0.3-2.0 \mathrm{keV}$ and $2.0-10.0 \mathrm{keV}$ bands. Given the multiple X-ray sources in the Swift/XRT field we extracted source events from a $40^{\prime \prime}$ circular region, and background events from a combination of background regions from areas free from other sources. To account for the high background and the

\footnotetext{
1 http://www.swift.ac.uk/user_objects/
}

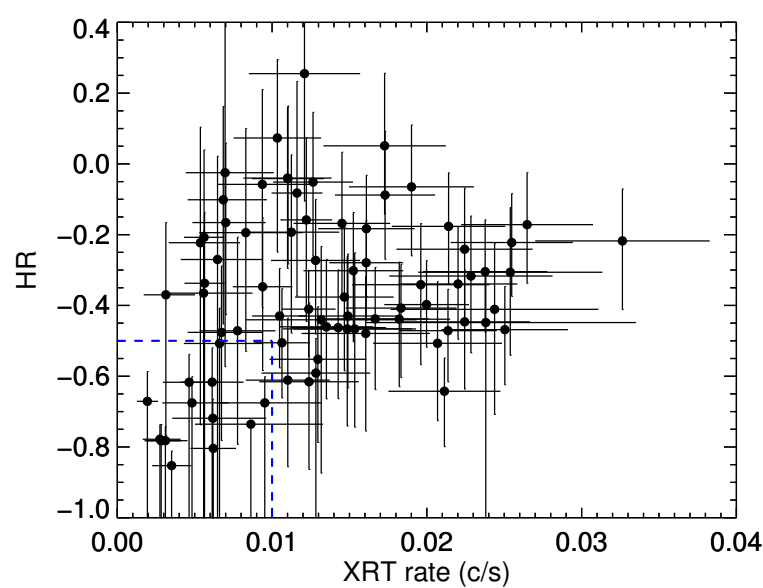

Figure 3. Spectral evolution of M51 ULX-7 as a function of the detected Swift/XRT count rate. Low count rates are consistent with a super-soft spectrum (i.e. region marked with dashed blue lines).

low number of source events during many observations we used a Bayesian estimator of HR (Park et al. 2006) that is publicly available as a command line program ${ }^{2}$.

For the constructed Swift/XRT light curve we computed the Lomb-Scargle (LS) periodogram ${ }^{3}$ (Scargle 1982; Horne \& Baliunas 1986; VanderPlas 2018). When using the complete Swift/XRT light-curve we detected a periodic signal ( $\sim 38.58 \mathrm{~d})$. A similar period $(\sim 38.87 \pm 0.20 \mathrm{~d})$ with higher significance was derived when we used only the data from Epoch II. While the LS periodogram did dot yield any significant detection when using only data from Epoch I, the highest periodogram peak was found at $\sim 48.7 \mathrm{~d}$. Given

\footnotetext{
2 hea-www.harvard.edu/astrostat/BEHR/index.html

3 Python code available at: https://github.com/jakevdp/ PracticalLombScargle/
} 
the Swift/XRT coverage within the first epoch, the $48.7 \mathrm{~d}$ period is quite uncertain, and we can only refer to this as an indication for a change in the super-orbital periodicity.

The X-ray light-curve of M51 ULX-7 is presented in Fig. 1. On the same figure we overplotted sinusoidal curves with a period measured by the LS periodogram. The LS periodogram for the 2 epochs is plotted in Fig. 2. The computed HRs are plotted as a function of Swift/XRT count rate in Fig. 3.

Based on our analysis, we report on the presence of a quasi periodic signal in the X-ray light curve of M51 ULX-7. The period of modulation is $\sim 39 \mathrm{~d}$, however there is some indication that the period can change and be as high as $\sim 49 \mathrm{~d}$. During the low luminosity intervals the system seems to transit to a super-soft state where only emission from a soft thermal component is detected in deep $X M M$-Newton observations. The estimated HRs support this claim as $\mathrm{HR} \rightarrow-1$ for low count rates.

\section{NEUTRON STAR SPIN-UP FROM ACCRETION}

\subsection{Basic equations}

Accretion theory has been the subject of numerous studies since the discovery of the first X-ray pulsars. For completeness and introducing the appropriate notations we will summarize the equations needed to constrain the magnetic field of the NS (i.e. at polar surface $B$ ) from basic observable quantities. This will also help to remind the reader some of the basic assumptions in our treatment.

For an accreting object the Eddington luminosity is obtained by equating the outward radiation pressure with the gravitational force:

$L_{\mathrm{Edd}} \approx 1.5 \times 10^{38} \mathrm{~m}_{1} \mathrm{erg} \mathrm{s}^{-1}$,

where $m_{1} \equiv M_{\mathrm{NS}} / M_{\odot}$ is the NS mass (in units of the solar mass). At low mass accretion rates, the accretion disc around the compact object is locally sub-Eddington and its inner part is gas-pressure dominated (e.g. see Mönkkönen et al. 2019). For mass accretion rates exceeding the critical rate $\dot{M}_{\text {Edd }}$, part of the dissipated energy is used to launch mass outflows from the inner part of the accretion disc, thus leading to a reduced accretion rate onto the compact object. This critical luminosity is defined as:

$\dot{M}_{\text {Edd }} \approx 2 \times 10^{18} \mathrm{~m}_{1} \mathrm{~g} \mathrm{~s}^{-1}$,

Outflows are launched inside the spherization radius $R_{\mathrm{sph}}$, where the disc thickness becomes comparable to its radius (Shakura $\&$ Sunyaev 1973) or, equivalently, the disc luminosity becomes equal to $L_{\mathrm{Edd}}$ (see eq. 18 in Poutanen et al. 2007):

$R_{\mathrm{sph}} \approx 10 \frac{G M_{\mathrm{NS}} \dot{m}_{0}}{c^{2}} \simeq 15 m_{1} \dot{m}_{0} \mathrm{~km}$,

where $G$ is the gravitational constant, and $\dot{m}_{0}$ is the mass accretion rate at $R_{\text {sph }}$ in units of $\dot{M}_{\text {Edd }}$. In a simple way, outflows are re-configuring accretion in a way that the local disc accretion rate is sub-Eddington, and the mass accretion rate at $R<R_{\text {sph }}$ can be written as (Shakura \& Sunyaev 1973):

$\dot{M}(R) \simeq \frac{R}{R_{\text {sph }}} \dot{m}_{0} \dot{M}_{\text {Edd }}$.

This approximation does not take into account the effects of heat advection in the disc. The latter results in a less abrupt decrease of $\dot{M}$ with radius than the one dictated by eq. (4) (Poutanen et al. 2007; Chashkina et al. 2019; Mushtukov et al. 2019).

For magnetized NSs the accretion disc does not extend near the NS, but is truncated at much larger radii due to the interaction with the NS magnetosphere. The magnetospheric radius provides an estimate of the disc inner radius (Ghosh et al. 1977):

$R_{\mathrm{M}}=\xi\left(\frac{R_{\mathrm{NS}}^{12} B^{4}}{2 G M_{\mathrm{NS}} \dot{M}^{2}}\right)^{1 / 7}$,

where $R_{\mathrm{NS}}$ is the neutron star radius and $\xi \sim 0.5$ (Campana et al. 2018). For typical $B$ values in X-ray pulsars (e.g., $10^{12} \mathrm{G}$ ), very high mass accretion rates are required (e.g., $\left.\dot{m}_{0}>10\right)$ to make $R_{\text {sph }}>$ $R_{\mathrm{M}}$. Moreover, as we will discuss in the following sections, the $\xi$ parameter could change as the mass accretion rate becomes superEddington, due to changes in the disc structure and conditions (see for details: Chashkina et al. 2019) .

An important condition in order for accretion to occur is that the Keplerian disc should be truncated inside the corotation radius:

$R_{\mathrm{co}}=\left(\frac{G M_{\mathrm{NS}} P_{\mathrm{NS}}^{2}}{4 \pi^{2}}\right)^{1 / 3}$.

Let us now consider the interaction of the in-falling matter with the NS. As material is transferred from the inner disc radius to the $\mathrm{NS}$, it also transfers angular momentum. The applied torque due to mass accretion is proportional to $\dot{M}_{\mathrm{NS}}$ (same as $\dot{M}\left(R_{\mathrm{M}}\right)$ ) and $\sqrt{R_{\mathrm{M}}}$, as it acts like a lever arm onto the star (i.e. $N_{\text {acc }} \approx \dot{M} \sqrt{G M R_{\mathrm{M}}}$ ). An additional torque term acts due to coupling of magnetic field lines and the disc (Ghosh \& Lamb 1979b). The total torque can be expressed in the form of $N_{\text {tot }}=n\left(\omega_{\text {fast }}\right) N_{\text {acc }}$ where $n\left(\omega_{\text {fast }}\right)$ is a dimensionless function, and $\omega_{\text {fast }}=\left(R_{\mathrm{M}} / R_{\mathrm{co}}\right)^{3 / 2}$ is known as the fastness parameter. Wang (1995) has provided an analytic relation for expressing this dimensionless function:

$n\left(\omega_{\text {fast }}\right)=\frac{7 / 6-(4 / 3) \omega_{\text {fast }}+(1 / 9) \omega_{\text {fast }}^{2}}{1-\omega_{\text {fast }}}$.

Based on eq. 7, the induced torque onto the NS goes to zero as $\omega_{\text {fast }} \rightarrow 0.95$, and thus for $\omega_{\text {fast }}>0.95$ the NS will spin down ${ }^{4}$. For $R_{\mathrm{M}} \simeq R_{\text {co }}$, the NS will spin down as the negative torque from interaction of the magnetic field lines with the accretion disc (that rotates slower than the NS) will dominate. For slow rotators (i.e. $R_{\mathrm{M}}<<$ $R_{\text {co }}$ ) a valid approximation is $n\left(\omega_{\text {fast }}\right)=7 / 6$. The change of the frequency of the NS is then given by:

$\dot{v}=\frac{n\left(\omega_{\mathrm{fast}}\right)}{2 \pi I_{\mathrm{NS}}} \dot{M}\left(\mathrm{R}_{\mathrm{M}}\right) \sqrt{G M_{\mathrm{NS}} R_{\mathrm{M}}}$,

where $I_{\mathrm{NS}} \simeq(1.0-1.7) \times 10^{45} \mathrm{~g} \mathrm{~cm}^{2}$ is the moment of inertia of the NS (e.g., Steiner et al. 2015). Equations 6 and 8, assume angular momentum is transferred by a Keplerian disc. It has been speculated that the induced torque might be suppressed by radiationpressure supported sub-Keplerian discs (Andersson et al. 2005). However, detailed calculations have shown that non-Keplerianity should only affect the disc rotation by less than $10 \%$ for typical parameters of PULXs (Chashkina et al. 2017).

The above equations are needed to probe the physical properties of a ULX system from basic observational quantities, like the spin-up rate and the observed $L_{\mathrm{X}}$. The measured $L_{\mathrm{X} \text {,iso }}$ of the system, can be converted to a mass accretion rate $\dot{M}$ assuming some efficiency. This is assumed to be the efficiency with which gravitational energy is converted to radiation $L_{\mathrm{X} \text {,iso }}=G M_{\mathrm{NS}} \dot{M} / R$. For $R=R_{\mathrm{NS}}=10^{6} \mathrm{~cm}$ and $M_{\mathrm{NS}}=1.4 M_{\odot}$, we find $L_{\mathrm{X}} \approx 0.2 \dot{M} c^{2}$. How-

4 We refer the reader to the recent work of Parfrey et al. (2016) for more details and a comparison between different regimes and solutions. 
Table 1. Properties of M51 ULX-7

\begin{tabular}{|c|c|c|c|c|}
\hline parameter & \multicolumn{3}{|c|}{ Values } & \multirow[t]{2}{*}{ Units } \\
\hline $\mathrm{b}$ factor ${ }^{(a)}$ & 1 & 4 & 12 & \\
\hline$\dot{M}_{\mathrm{NS}}$ & 14.1 & 3.5 & 1.2 & $\dot{M}_{\text {Edd }}$ \\
\hline$R_{\mathrm{sph}}^{(b)}$ & 296 & 74 & 25 & $\mathrm{~km}$ \\
\hline \multicolumn{5}{|c|}{ Secular spin-up ${ }^{(c)}$} \\
\hline$B$ & $3 \times 10^{11}$ & $1.9 \times 10^{12}$ & $5.2 \times 10^{14}$ & $\mathrm{G}$ \\
\hline$R_{\mathrm{M}}$ & 140 & 2200 & 20000 & $\mathrm{~km}$ \\
\hline \multicolumn{5}{|c|}{ Measured spin-up ${ }^{(d)}$} \\
\hline$B$ & $1.3 \times 10^{8}$ & $8.6 \times 10^{9}$ & $2.3 \times 10^{11}$ & $\mathrm{G}$ \\
\hline$R_{\mathrm{M}}$ & 1.7 & 28 & 248 & $\mathrm{~km}$ \\
\hline \multicolumn{5}{|c|}{ Comparison to other work ${ }^{(e)}$} \\
\hline$B_{\mathrm{AC}}$ & $8.5 \times 10^{13}$ & $1.2 \times 10^{13}$ & $8.5 \times 10^{11}$ & G \\
\hline$R_{\mathrm{M}}$ & 3500 & 1700 & 520 & $\mathrm{~km}$ \\
\hline$\dot{v}$ & $1.4 \times 10^{-9}$ & $2.5 \times 10^{-10}$ & $4.6 \times 10^{-11}$ & $\mathrm{~Hz} / \mathrm{s}$ \\
\hline
\end{tabular}

(a) Beaming factor used to translate the observed maximum $L_{X}=7.1 \times 10^{39}$ $\mathrm{erg} / \mathrm{s}^{2}$ to intrinsic luminosity and accretion rate. (b) $R_{\text {sph }}$ was estimated assuming no outflows; i.e $\dot{M}_{\mathrm{NS}}$ is the mass accretion rate of the disc. ${ }^{(c)}$ The magnetic field was estimated based on the maximum observed secular spinup rate; $\dot{v}=2.8 \times 10^{-10} \mathrm{~Hz} / \mathrm{s}$ (see text). ${ }^{(\mathrm{d})}$ The magnetic field was estimated from eq. 10, based on the observed spin-up rate from individual observations $\dot{v}=3.1 \times 10^{-11} \mathrm{~Hz} / \mathrm{s}$ (see text). ${ }^{\text {(e) }}$ Rodríguez Castillo et al. (2019) have compared the maximum observed $L_{X}$ for a given beaming factor with the theoretical model of Mushtukov et al. (2015) in order to derive the quoted $B$ values.

ever, in ULX systems, a low to moderate amount of beaming can be present due to the presence of outflows (King et al. 2017). Thus the intrinsic luminosity $\left(L_{\mathrm{X}}\right)$ of the system can be expressed as a function of beaming parameter $b$ (i.e. $L_{\mathrm{X}}=L_{\mathrm{X} \text {,iso }} / b$ ):

$\frac{L_{\mathrm{X}, \text { iso }}}{b}=\frac{G M_{\mathrm{NS}} \dot{M}}{R}$

To put the above into perspective, it is crucial to consider all above conditions and equations when probing the properties of M51 ULX-7. For example, if we invoke beaming in order to explain the observed super-Eddington luminosity, the derived mass accretion rate and $B$ should yield an inner disc radius smaller than $R_{\text {sph }}$, as this is a necessary condition to have outflows and beaming (Poutanen et al. 2007).

\subsection{NS magnetic field from spin-up}

Having laid out the framework for estimating the NS magnetic field, we can proceed with an application to M51 ULX-7. Analysis of archival XMM-Newton data showed two periods with very different secular spin evolution (Rodríguez Castillo et al. 2019). According to the authors, between MJD 53552 and MJD 55723 the spin period of the NS evolved from 3.2831(2) s to 2.8014(7) s, yielding a secular spin-up of $\dot{P}=-2.57 \times 10^{-9} \mathrm{~s} / \mathrm{s}$ (or $\dot{v}=2.8 \times 10^{-10} \mathrm{~Hz} / \mathrm{s}$ ). Between MJD 55723 and MJD 58284 the spin period of the NS evolved from 2.8014(7) to 2.7977148(2) s, yielding a secular spinup of $\dot{P}_{\text {sec }}=-1.67 \times 10^{-11} \mathrm{~s} / \mathrm{s}\left(\right.$ or $\left.\dot{v}_{\mathrm{sec}}==2.13 \times 10^{-12} \mathrm{~Hz} / \mathrm{s}\right)$. The spin-up of the system can also be constrained by using accelerated epoch folding. This was only feasible from the combination of two $X M M$-Newton observations (span of 2 days), and was determined to be $\dot{P}=-2.4 \times 10^{-10} \mathrm{~s} / \mathrm{s}\left(\right.$ or $\left.\dot{v}=3.1 \times 10^{-11} \mathrm{~Hz} / \mathrm{s}\right)$ on MJD 58283.44. The latter $\dot{P}$, is calculated by taking into account the orbital motion of the binary (Rodríguez Castillo et al. 2019). On the other hand, the secular spin-up is affected by Doppler shifts due to orbital motion. However, the effect of orbital modulation in the observed $\mathrm{P}$ value only has an amplitude of $\sim 0.006 \mathrm{~s}$, thus the estimated $\dot{P}_{\text {sec }}$ is only affected by $<2 \%$. The estimation of $B$ must be based on the simultaneous measurements of $L_{\mathrm{X}}$ and $\dot{v}$. Nevertheless, constraints can also be made by using the secular spin-up rate of M51 ULX-7, under certain assumptions.

Assuming M51 ULX-7 is at the slow rotator regime $\left(R_{\mathrm{M}}<<\right.$ $R_{\text {co }}$ ) and the NS away from spin equilibrium, we can estimate $B$ at the NS surface and $\dot{M}$ onto the NS. For our calculations we adopt $\xi=0.5, R_{\mathrm{NS}}=10^{6} \mathrm{~km}, M_{\mathrm{NS}}=1.4 M_{\odot}, I_{\mathrm{NS}}=1.3 \times 10^{45} \mathrm{~g} \mathrm{~cm}^{-2}$. By solving eq. 5, 8, 9 for the polar magnetic field strength:

$B \simeq 740 n\left(\omega_{\text {fast }}\right)^{-7 / 2} \xi^{-7 / 4} G^{3 / 2} M_{\mathrm{NS}}^{3 / 2} R_{\mathrm{NS}}^{-6} I_{\mathrm{NS}}^{7 / 2} \dot{v}^{7 / 2}\left(L_{\mathrm{X}, \text { iso }} / b\right)^{-3} G$,

where $L_{\mathrm{X} \text {,iso }}$ is the observed luminosity, and $\dot{v}$ the observed spin-up rate. Then by using eq. 2, 3, 5, 9, we can estimate the $R_{\mathrm{M}}$ and $R_{\text {sph }}$ for different beaming factors. Our numerical calculations are based on the Wang (1995) approximation (see eq. 7) and are presented in Table 1. Our results can be compared to those of Rodríguez Castillo et al. (2019). In their work the authors assumed that the maximum observed $L_{\mathrm{X}}$ from the system is related to the surface magnetic field strength of NS based on theoretical predictions; i.e. $L_{39} \approx 0.35 B_{12}^{3 / 4}$ (Mushtukov et al. 2015). For convenience we refer to the theoretical predicted magnetic field of the accretion column as $B_{\mathrm{AC}}$.

From the values in Table 1, it is clear that no combination of parameters can self-consistently describe the observed values of M51 ULX-7. Specifically, by assuming no beaming, the estimated $B$ value is too small to explain the high luminosity from the accretion column (Mushtukov et al. 2015). In addition, the intrinsic spin up rate yields inner disc radii consistent with the NS size, thus this solution is highly improbable. On the other hand, a moderate beaming (i.e. 4-12) yields a size for the inner disc much larger than the $R_{\text {sph }}$, i.e. is in contrast with the conditions needed for beaming. Finally, in some cases the estimated $R_{\mathrm{M}}$ is larger than the corotation radius of the system $\left(R_{\mathrm{co}} \sim 3300 \mathrm{~km}\right)$, thus indicating that our assumption for being at the slow rotator regime is not met. Therefore, at least some of our original assumptions behind this approach must be incorrect. For example the structure of the disc in these high accretion rates should affect our estimations, or perhaps the system is not in the slow rotator regime.

\subsubsection{Comparison with semi-analytical models}

The analytical estimations of the previous section rely heavily on the assumption of a thin disc. Moreover, $\xi$ is known to vary from the default value of 0.5 , for discs accreting at super-Eddington rates. In these section we compare the observed properties of M51 ULX-7 with the semi-analytical calculations of Chashkina et al. (2019). In their recent work the authors have computed the inner radius of the accretion disc as a function of accretion rate for various $B$ values. These theoretical predictions are plotted in Fig. 4. From Fig. 4 it is clear that for $B>10^{14} G$ a mass accretion rate larger than $10 \dot{M}_{\mathrm{Edd}}$ is required for the system to be in the accretor regime. Moreover, for outflows and thus beaming to occur, the mass accretion at the inner disc radius (thus in the NS too: $\dot{M}_{\mathrm{NS}}$ ) should be larger than $10 \dot{M}_{\mathrm{Edd}}$ even for a moderate magnetic field strength $10^{12} \mathrm{G}$.

A comparison of the inner disc radius as estimated by the semi-analytical model of Chashkina et al. (2019) with the classical prescription provided by eq. 5 can be used to provide an order of magnitude estimate for the uncertainties introduced by adopting the classical relation. For example there is always a specific $\dot{M}$ value where the two prescriptions provide the same result (see Fig. 


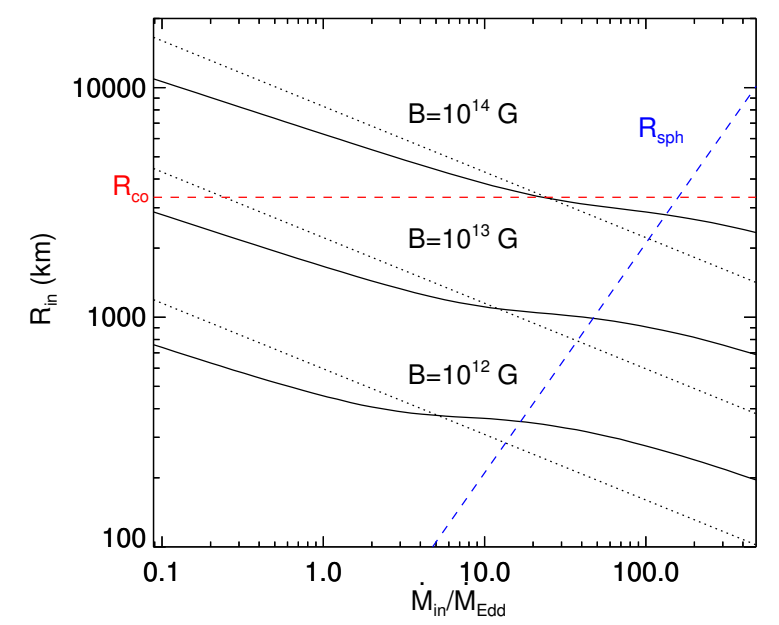

Figure 4. Theoretical prediction of the dependence of the inner disc radius on the accretion rate at the inner disc (Chashkina et al. 2019). Standard solutions (i.e. $R_{\text {in }} \propto \dot{M}_{\text {in }}^{-2 / 7}$ ) for $\xi=0.5$ are plotted with dotted lines. Blue dashed line follows $R_{\mathrm{sph}}$, and horizontal red dashed line marks $R_{\mathrm{co}}$.

4). For smaller (larger) $\dot{M}$ values the semi-analytical estimations yield smaller (larger) $R_{\text {in }}$ values for the disc. This difference can in general be no greater than a factor of 2 for typical $\dot{M}$ and $B$ values of PULXs.

By using eqs. 7 and 8 we can compute the predicted NS frequency derivative and compare it with the observed values (see Fig. $5)$. Given the spin of the NS ( 2.8 s), the system should be in the slow rotator regime, for any $B<5 \times 10^{13} G$ and for a range of mass accretion rates larger than $0.1 \dot{M}_{\mathrm{Edd}}$. However, for $B=10^{14} G$ the total torque should be zero for $\dot{M}_{\text {in }} \sim 30 \dot{M}_{\text {Edd }}$.

The large variations of observed secular $\dot{v}$ is also in favor of excluding solutions with low magnetic field (see Fig. 5). For low $B$ the system should always remain in the slow accretor regime and variations of $\dot{v}$ by two orders of magnitude do not agree with mass transfer through Roche Lobe overflow (Rodríguez Castillo et al. 2019), that should be relatively stable. We conclude that the observed properties of M51 ULX-7 cannot be adequately explained assuming a NS accreting in the slow accretor regime, even by using a more realistic disc structure compared to the standard thin disc.

Thus, following Occam's Razor the NS should be rotating near equilibrium, where relatively small changes in $\dot{M}_{\mathrm{NS}}$ should result in large changes in the induced torque. For an equilibrium period of $P_{\mathrm{NS}}=2.8 \mathrm{~s}$ and by following eq. 7 we get a magnetic field of $4-7 \times 10^{13} \mathrm{G}$ assuming a beaming factor of $1-4$.

\subsection{Uncertainty of $B$ based on different torque models}

The estimated $B$ value is affected by our poor knowledge of accretion torque models. In the literature there are several torque models adopted for X-ray pulsars; i.e. Ghosh \& Lamb (1979a), Wang (1995), Rappaport et al. (2004). Although most of them are in reasonable agreement for slow rotators, there can be a large discrepancy for the estimate of the equilibrium period. Analytically, this discrepancy lies in the derivation of $n\left(\omega_{\text {fast }}\right)$ that becomes zero for different $\omega_{\text {fast }}$ values. Following Wang (1995) the equilibrium period is found for $R_{\mathrm{M}} / R_{\mathrm{co}} \sim 0.95$, while from the earlier prescription of Ghosh \& Lamb (1979a) we get $R_{\mathrm{M}} / R_{\mathrm{co}} \sim 0.5$. Rappaport et al. (2004) focused in the case where $R_{\mathrm{M}}>R_{\mathrm{co}}$, and argued that in this case the disc re-configures such as $R_{\text {in }} \sim R_{\text {co }}$, while for cases

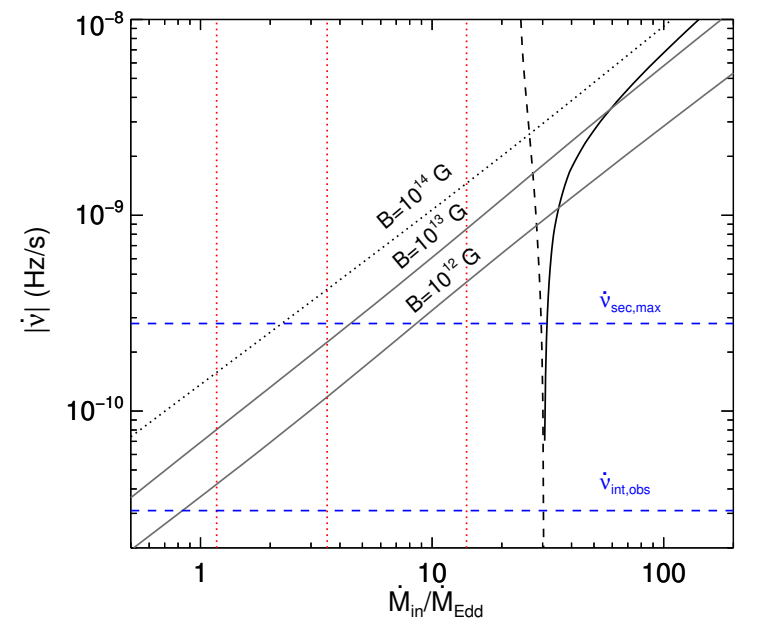

Figure 5. Theoretical prediction of the induced spin-up of the NS as a function of the accretion rate at the inner disc (Chashkina et al. 2019). Blue dashed line marks the observed maximum secular $\dot{v}$, and vertical red dotted line marks the calculated $\dot{M}_{\mathrm{NS}}$ for a beaming factor of 12,4 and 1 (i.e. no beaming) from left to right. In our calculation we have defined $n\left(\omega_{\text {fast }}\right)$ as in eq. 7. For magnetic field strengths of $10^{12}-10^{13} \mathrm{G}$, the system remains in the slow rotator regime for the range of accretion rates used in the plot. For $B=10^{14} \mathrm{G}$, spin equilibrium is reached for $\dot{M}_{\text {in }} \sim 30 \dot{M}_{\text {Edd }}$. For clarity we used solid and dotted lines to denote the spin-up and spin-down regimes respectively. Finally, with the dotted black line we plotted the expected spinup rate if $\omega_{\text {fast }}=0$ for $B=10^{14} \mathrm{G}$.

where $R_{\text {in }}<R_{\text {co }}$ their solution is in reasonable agreement with the one by Wang (1995) (see also Parfrey et al. 2016, and their Fig. 2 for a comparison). Based on Ghosh \& Lamb (1979a) prescription, and assuming 2.8 is the equilibrium period of M51 ULX-7, we get $B \sim 2.3 \times 10^{13} \mathrm{G}$ for the NS, or a factor of 3 lower value from the one derived using the Wang (1995) prescription.

Another mechanism that can potentially affect the spin equilibrium of the NS is the enhancement of spin-down torques from disc induced opening of the pulsar magnetic field lines (Parfrey et al. 2016). This mechanism has been used in order to naturally explain millisecond pulsars with spin periods larger than $1 \mathrm{~ms}$, although classic torque theory would expect them to rotate at much higher rates. The opening of field lines could be responsible for enhancing the spin-down torque of the pulsar wind by a factor of $\left(R_{\mathrm{LC}} / R_{\mathrm{M}}\right)^{2}$, where $R_{\mathrm{LC}}$ is the light-cylinder of the pulsar. The spindown torque is thus given by (see eq. 18 of Parfrey et al. 2016):

$N_{\text {down,open }}=\frac{\left(B^{2} R_{\mathrm{NS}}^{3}\right)^{2}}{R_{\mathrm{M}}^{2}} \frac{2 \pi}{c P}$,

where we have assumed maximum efficiency for the magnetic field opening. Although this model is a decent approximation for the most rapid systems, for PULXs systems with high $\dot{M}$ the model predicts very high $B$ values, and yields equilibrium conditions that require $\left(R_{\mathrm{co}}<R_{\mathrm{M}}\right)^{2}$. In physical terms, PULXs enter the propeller regime before this spin-down term becomes dominant. Thus, this mechanism alone could not be responsible for M51 ULX-7 being at equilibrium. For the case of M51 ULX-7, assuming all magnetic field lines between $R_{\mathrm{LC}}$ and $R_{\mathrm{M}}$ open, then the induced torques due to the enhanced pulsar wind would only equal to $20-25 \%$ of the torque due to accretion (see eq. 18 of Parfrey et al. 2016). As a result, it would effectively change the shape of the cusplike struc- 


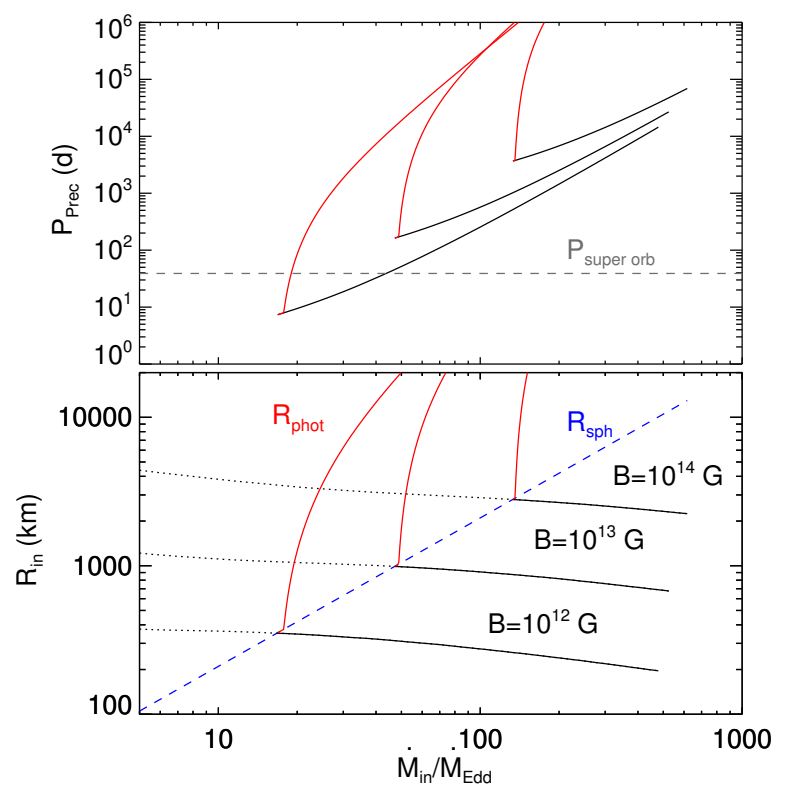

Figure 6. Lower panel: Theoretical prediction of the inner disc radius as a function of accretion rate for various magnetic field strengths (same as Fig. 4). A necessary condition for the presence of outflows is that the inner disc radius is smaller than $R_{\text {sph }}$ (blue dashed line). The maximum extent of the outflows is given by the photospheric radius $R_{\text {phot }}$ (red lines). Upper panel: The precession period of the outflows can be estimated based on the model of Middleton et al. (2018b). A lower limit for the extent of the outflows, and thus for the precession period $P_{\mathrm{Prec}}$, can be given by $R_{\mathrm{sph}}$ (black lines); an upper limit for $P_{\text {Prec }}$ (red lines) is obtained by assuming that the system precesses as a solid body up to a distance of $R_{\text {phot }}$.

ture in the $\dot{M}-|\dot{v}|$ diagram (i.e. broaden it), and it would decrease the required magnetic field strength for making M51 ULX-7 to be at equilibrium by $\lesssim 3 \%$.

To summarise, assuming no beaming in M51 ULX-7 observed flux, uncertainties induced by our poor knowledge of torque models would yield a magnetic field strength of the NS of the order of $2-7 \times 10^{13} \mathrm{G}$ for an equilibrium period of $2.8 \mathrm{~s}$.

\section{NATURE OF THE SUPER-ORBITAL MODULATION}

The detection of a quasi-periodic super-orbital modulation in the X-ray flux of M51 ULX-7 is perhaps a probe of precession from the disc. Let us thus consider some basic mechanisms that could be responsible for such precession.

\subsection{Lense-Thirring precession}

The effects of Lense-Thirring precession (Bardeen \& Petterson 1975) have recently been discussed in the context of ULXs (Middleton et al. 2018b, 2019), in order to explain their super-orbital modulation. Moreover, it has been proposed to explain the rapid change of the radio jet orientation of V404 Cygni during its 2015 outburst (Miller-Jones et al. 2019). According to Middleton et al. (2018b), the precession period depends on parameters such as the NS spin, the size of $R_{\text {sph }}$ and the extent of the outflows $\left(R_{\text {out }}\right)$. Fol- lowing Middleton et al. (2018b) the precession timescale is:

$P_{\mathrm{prec}} \approx P_{\mathrm{NS}} \frac{R_{\mathrm{sph}}^{3} c^{2}}{6 G I_{\mathrm{NS}}} \frac{1-\left(R_{\mathrm{M}} / R_{\mathrm{sph}}\right)^{3}}{\ln \left(R_{\mathrm{sph}} / R_{\mathrm{M}}\right)}\left(\frac{R_{\mathrm{out}}}{R_{\mathrm{sph}}}\right)^{2}$,

An upper limit for the extent of the outflows can be estimated by estimating the distance at which the optical depth becomes less than one (i.e. photospheric radius $\mathrm{R}_{\text {phot }}$ ), while a lower limit is given by $\mathrm{R}_{\text {sph }}$. The Middleton et al. (2018b) model has been also used to explain the observed properties of NGC300 ULX1 (Vasilopoulos et al. 2019). Vasilopoulos et al. (2019) found that although the observed flux from NGC300 ULX1 changed by a factor of $\sim 50$ within a few months, the spin up of the system continued at a steady pace (constant $\dot{v}$ ). Based on the observed properties of NGC300 ULX1 one can expect a timescale of precession of the order of a year, thus naturally explaining extended epochs of decreased flux due to obscuration from the outflows.

We can estimate the timescale of precession for M51 ULX7 by following Middleton et al. (2018b) and invoking the same analytical relations used for NGC300 ULX1 (see eq. 13 \& 14 of Vasilopoulos et al. 2019). In Fig. 6 we plot the predicted precession period as a function of mass accretion rate, for various values of $B$. A timescale of precession similar to the observed one (i.e. 40 d), is only possible for super-Eddington accretion, and magnetic field strength lower than a few times $10^{12} \mathrm{G}$. However, this configuration requires M51 ULX-7 to be in the slow-rotator regime and would yield a much higher NS spin-up rate than the observed one (see Fig. 5).

\subsection{Precession due to the magnetic dipole torques}

The interaction of the disc and the magnetosphere is a complicated process that is not fully understood, but can lead to different quasiperiodic phenomena (Lai 1999). The viewing angle of the disc can change on time-scales of months-years due to the interactions between the magnetic field and the disc (Lipunov \& Shakura 1980). The spin-averaged torques by the dipole magnetic field lead to the inner twisted parts of the disc taking on a spiral-like shape with a certain direction of twisting. The forces applied to the disc will tend to twist it along the magnetic axis into a stable position. The precession period is then given by:

$$
\begin{aligned}
P_{\text {prec,mag }} \approx & 1.5 \times 10^{4}\left(\frac{B}{10^{12} \mathrm{G}}\right)^{-2}\left(\frac{R_{\mathrm{NS}}}{10^{6} \mathrm{~cm}}\right)^{-2}\left(\frac{R_{\mathrm{M}}}{10^{8} \mathrm{~cm}}\right)^{3}\left(\frac{P_{\mathrm{NS}}}{1 \mathrm{~s}}\right)^{-1} \\
& \left(\frac{I_{\mathrm{NS}}}{10^{45} \mathrm{gr} \mathrm{cm}^{2}}\right) \frac{1}{\cos \psi(3 \cos \zeta-1)} \mathrm{yr},
\end{aligned}
$$

where $\psi$ is the angle between the NS spin axis and the normal of the accretion disc, $\zeta$ is the angle between the spin axis and magnetic field axis. Although this process has been proposed to explain the precession of PULXs (Mushtukov et al. 2017), it would require extremely high (dipole) magnetic field values and mass accretion rates to reproduce the observed super orbital periods in PULXs (i.e. $P_{\text {prec,mag }} \propto B^{-2 / 7} \dot{M}^{-6 / 7}$ ). For M51 ULX-7 it would require mass accretion with a rate larger than $1000 \dot{M}_{\text {Edd }}$ and $B$ higher than $10^{15}$ G. Thus a clock related to this mechanism would beat at much lower frequency than the observed super-orbital modulation.

\subsection{NS free precession}

As we already mentioned, it is worth noting the similarities of M51 ULX-7 with Her X-1, one of the first discovered X-ray pul- 
sars. Her X-1 $(P=1.24 \mathrm{~s})$ has an orbital period of $1.7 \mathrm{~d}$, and a super-orbital period of $\sim 35 \mathrm{~d}$ (Tananbaum et al. 1972; Katz 1973). The nature of the super-orbital modulation has been speculated to to arise by precession of the companion star and a twisted accretion disc (Petterson 1975), while a modulation of the pulse profile with super-orbital phase is also an indication of precession of the NS itself (Truemper et al. 1986). NS free precession has also been invoked in order to explain long term periodic changes from observations of isolated NSs: both in their spectral properties (e.g. RX J0720.4-3125 Haberl et al. 2006) and in the longtimescale sinusoidal radio-timing residuals from a few sources (e.g. PSR B1828-11 Stairs et al. 2000).

Free precession does not necessarily act alone in any of these systems, however. For the case of Her X-1 it has since been argued that there are two different clocks that are responsible for its super orbital periodicities, and which are generally synchronized (Staubert et al. 2009). For the radio pulsar PSR B1828-11, the discovery of abrupt pulse-profile changes indicated the star's magnetosphere was switching between two different spin-down states and has led some to discard the precession model, since the apparently smooth slow oscillation of the residuals shows as discrete sharp steps on shorter timescales (Stairs et al. 2019). On the other hand, the long-term average of these steps is so periodic that it seems that there must be some mechanism setting this periodicity - and a freely-precessing NS could plausibly be such a clock (Jones 2012).

In a similar way, the super-orbital periodicity of M51 ULX-7 could be driven by free precession of the system's NS, even though we found indications that this period varies somewhat. Within this paradigm, assuming the possible accretion-disc precession to be synchronised with that of the NS, we can obtain useful constraints on any distortion $\epsilon$ of the star that is misaligned from the rotation axis (the motion is essentially independent of the axisymmetric centrifugal bulge of the star). This distortion may be either elastic or magnetic in origin. Elastic distortions will be confined to the star's crust, although modelling of their size and structure is fraught with uncertainties, as they will depend on the star's seismic history; they could even be negligible. The star's internal magnetic field is a more reliable source of distortion, since a magnetic field always deforms its host star, with a predictable size scaling with the ratio of the magnetic energy to the gravitational binding energy (Chandrasekhar \& Fermi 1953).

Following (Jones \& Andersson 2001) we get:

$\epsilon=\frac{P_{\mathrm{NS}}}{P_{\text {prec }, \text { obs }} \cos \chi} \simeq 7.72 \times 10^{-7} \frac{P_{\mathrm{NS}}}{1 \mathrm{~s}}\left(\frac{P_{\text {prec }, \mathrm{obs}}}{30 \mathrm{~d}} \frac{\cos \chi}{0.5}\right)^{-1}$,

where $\chi$ is the angle of the NS misaligned distortion compared to its rotation axis. For the observed parameters of the system we deduce $\epsilon=(0.83-1.2) \times 10^{-6}$ for the range ${ }^{5} \chi=0-45^{\circ}$. Now assume all this distortion is due to magnetic effects, so that $\chi$ represents the misalignment between the star's rotation and magnetic-field axes (and is therefore equal to the angle $\zeta$ from equation (13)). Quantitative solutions for a simple fluid model of a magnetically-distorted NS (Lander \& Jones 2009) give:

$\epsilon=2 \times 10^{-11}\left(\frac{B_{\text {surf }}}{10^{12} \mathrm{G}}\right)^{2}$,

where the $B^{2}$ scaling comes from the form of the magnetic energy. This leads us to infer a rather strong magnetic field, $B_{\text {surf }} \approx 2 \times 10^{14}$ $\mathrm{G}$ for the full range of $\epsilon$ we consider; Postnov et al. (2013) ob-

${ }^{5}$ Note that equation (14) diverges in the limit $\chi \rightarrow 90^{\circ}$. tained a similar estimate for Her X-1. However, this model is somewhat unrealistic, as the presence of superconducting protons in the NS core changes the magnetic-energy scaling to be proportional to $B H_{\mathrm{c} 1}$, where $H_{\mathrm{c} 1}$ is a 'microscopic' magnetic field associated with the quantisation of magnetic flux on small scales. In practice, this leads to larger distortions for the same $B_{\text {surf }}$, compared with those obtained from a non-superconducting model like equation (15). The ellipticity relation now takes the form (Lander 2013, 2014):

$\epsilon=3 \times 10^{-8}\left(\frac{B_{\text {surf }}}{10^{12} \mathrm{G}}\right)\left(\frac{H_{\mathrm{cl}}(0)}{10^{16} \mathrm{G}}\right)$,

where $H_{\mathrm{cl}}(0)$ is the value of the (density-dependent) $H_{\mathrm{c} 1}$ at the centre of the star; we will set this to the sensible value of $10^{16} \mathrm{G}$. Using the above - more physically reasonable - ellipticity relation, our new estimate of the surface magnetic field is $B_{\text {surf }} \approx(3-4) \times 10^{13}$ $\mathrm{G}$ for $\chi=0-45^{\circ}$.

Even if the full dynamics of the ULX system are complex, a magnetically-distorted, freely-precessing NS is an attractive candidate for the central clock governing its super-orbital modulation. For the mechanism to work, the NS must couple with the disc in an effective manner - but the magnetospheric field lines provide a mechanism for this, tying the motion of charged particles in the disc with the NS on the Alfven crossing timescale of the system. Note, however, that free precession of the NS alone cannot account for the putative variation in super-orbital period of $39-49$ days.

\section{DISCUSSION}

Since the recent discovery of PULXs, the nature of their engines has been one of the major puzzles in high energy astrophysics. Highly magnetized NSs have been proposed as a mechanism for sustaining super-Eddington accretion (Mushtukov et al. 2015). However, constant mass transfer from super-giant companions might also be a necessary requirement (Lau et al. 2019). Thus, observational constraints on the NS magnetic field and mass transfer are needed to understand these extraordinary systems.

The recent detection of super-orbital flux modulation in PULXs (M82 X-2: 60 d, Kong et al. 2016; NGC 5907: 72 d, Walton et al. 2016; NGC 7793 P13: 65 d, Hu et al. 2017) has sparked renewed interest in this long-term periodic behavior, which is increasingly regarded as an indication for the presence of beamed emission in ULXs - the result of a funnel-like structure, generated by massive outflows. However, it is important to recall that longterm (10-100 d) periodic flux modulations (by a factor of $>10$ ), have long been detected in several normal X-ray pulsars with subEddington luminosities (e.g., Cyg X-2: Paul et al. 2000; Her X1: Katz 1973; LMC X-4: Naik \& Paul 2003; SMC X-1: BonnetBidaud \& van der Klis 1981). Therefore, it could be argued that this type of variability in X-ray binary systems is simply an indication for the presence of a high-B NS, rather than super-Eddington accretion.

Analysis of the Swift/XRT monitoring data of M51 ULX-7 revealed the presence of a $\sim 39 \mathrm{~d}$ super-orbital modulation. Our calculations suggests that the coupling of the NS free precession and the disk through the magnetospheric torques constitutes a plausible mechanism for this behavior, as has been demonstrated for other X-ray pulsars with similar characteristics (Postnov et al. 2013). We improve on previous modelling by accounting for the effect of superconductivity in the NS core on the precession period. An attractive feature of the free-precession model is that its inherent 
uncertainties (like the value of $\cos \chi$ and the exact model leading to equation (16)) only introduce order-unity changes, and - unlike Lense-Thirring precession - this model is independent of the complex magnetospheric physics of the system.

From the observational point of view, both Lense-Thirring and NS free precession timescales scale linearly with the NS spin. Thus, continuing monitoring of PULX systems like M51 ULX-7 could provide strong arguments in favor or against each mechanism. Given that Lense-Thirring precession also strongly depends on $\dot{M}$, the NS free precession scenario can be tested in a more straightforward way. The fact that most PULXs show changes in $\mathrm{P}$ on timescales of decades complicates such endeavor. Apart form M51 ULX-7, another potential candidate for such study might be the PULX NGC 300 ULX1, since in this particular system the NS is still at the slow rotator regime and its spin period has dramatically changed the last 4 years (from $\sim 124 \mathrm{~s}$ to $\sim 16 \mathrm{~s}$; see Vasilopoulos et al. 2018, 2019). However, in NGC 300 ULX1 no super-orbital period has been measured and given the system current properties $(\mathrm{P} \sim 16 \mathrm{~s})$ any periodicity due to precession might have timescales of years.

We must also note that several authors have proposed the precession of a radiation-driven warped disk as the origin of superorbital variability in X-ray binaries (e.g. Pringle 1996; Wijers \& Pringle 1999; Ogilvie \& Dubus 2001; Clarkson et al. 2003; Dage et al. 2019). This scenario - which has also been proposed to interpret similar temporal characteristics in ULXs M82 X-2 (Kong et al. 2016) and SS 433 (Foulkes et al. 2010) - cannot be ruled out for M51 ULX-7. Nevertheless, further investigation of the precessing warped disk mechanism as the engine behind the super-orbital modulation can be performed by smoothed particle hydrodynamic simulations and not by analytical or empirical relations. This is beyond the scope of this work and will be the focus of a future investigation.

The comparison of the observed properties of M51 ULX7 with theoretical models has demonstrated that strong beaming $(b>2)$ can be excluded, since a necessary condition should be the presence of outflows (i.e. $R_{\mathrm{M}}>R_{\mathrm{sph}}$; see Fig. 4). Moreover, although the NS exhibits a relative high secular spin-up rate, $|\dot{v}|$ is much smaller than would be expected for a system in the slow rotator regime (see Fig. 5). One obvious explanation is that the NS is rotating near equilibrium, in which case its magnetic field should be $2-7 \times 10^{13} \mathrm{G}$. This is in good agreement with the independent estimate of $B$ from NS free precession (see section 4.3). In the free-precession paradigm, the torque acting by the magnetosphere on the disc will change in phase with the NS precession. Although the coupling of the disc and NS precession period is a complicated process, free precession could be the clock behind the observed super-orbital modulation (Lai 1999; Postnov et al. 2013). In the case of an accretion disc precessing due to synchronization with the NS free precession, no strong outflows are required to explain the modulation in the X-ray light-curve.

An alternative scenario, to account for the low spin-up rate, would be that the NS rotation axis and the accretion disc plane are near-aligned, so that there is minimum angular momentum transfer from the disc to the NS. As the energy of the interaction of the disc and the magnetic dipole depends on their relative orientation, a perpendicular or parallel configuration between the rotation axis and the axis of the disc would provide a stable solution (Lipunov \& Shakura 1980). However, an application and confirmation of such models requires constraints on more modeled parameters - as the interactions between magnetized NSs and accretion discs are much more complicated- and a complete reference to different models would be beyond the scope of this work (see Lai 2014, 1999, and references within).

Attributing the $39 \mathrm{~d}$ modulation to disc precession can naturally explain the spectral changes observed with XMM-Newton (Rodríguez Castillo et al. 2019) and Swift/XRT monitoring (see Fig. 3 ). When the accretion disc is edge-on (low state) we only see the super-soft component from the disc and disc wind that create a photosphere (Zhou et al. 2019). Thus, during its low state M51 ULX7 is an analog to the ultraluminous super-soft X-ray sources like NGC 55 ULX (Pinto et al. 2017). Moreover, by invoking the NS free precession and a warped disc to explain the super orbital modulation in M51 ULX-7, it is not required the disc to be in the advection dominated regime, where outflows are expected (Chashkina et al. 2019; Mönkkönen et al. 2019; Mushtukov et al. 2019). Thus, $R_{\mathrm{M}}>R_{\text {sph }}$ is not a necessary condition for this phenomenon to take place. We note that even for the case where the $R_{\mathrm{M}}>R_{\text {sph }}$ a warping of the outer disc due to irradiation from the $\mathrm{AC}$ is quite likely to happen (Pringle 1996; Middleton et al. 2018b). However, the spectral properties during the low flux state (i.e. size and temperature of emitting region) are more compatible with emission from the innermost disk part. The soft emission originates from reprocessing of the beamed pulsar emission by a warped inner accretion disc that precesses with time (SMC X-1, Hickox \& Vrtilek 2005; LMC X-4, Hung et al. 2010; SMC X-3, Koliopanos \& Vasilopoulos 2018). In this picture the pulsar is covered by a shell of material (i.e. warped disk), while the covering fraction is the luminosity ratio of the soft to hard states $\Omega \sim L_{\text {soft }} / L_{\text {hard }} \sim 0.05$ (Hickox et al. 2004). An order of magnitude estimate of the size of the reprocessing region can be obtained by the temperature of the soft component observed in the low state (i.e. $0.19 \mathrm{keV}$ ) and the isotropic $L_{\mathrm{X}}$ of the pulsar as derived from the high state (i.e. $\sim 7 \times 10^{39} \mathrm{erg} \mathrm{s}^{-1}$ ) by following (Hickox et al. 2004):

$R_{\text {rep }}^{2}=\frac{L_{\mathrm{X}}}{4 \pi \sigma T_{\text {soft }}^{4}}$,

where $\sigma$ is the Stefan-Boltzmann constant. We then get $R_{\text {rep }} \sim$ $6000 \mathrm{~km}$, or about 2 times the corotation radius. This is more evidence in favor of the disc being truncated near corotation radius and thus the NS rotating near equilibrium period.

Finally, given the evidence of change in the period of the super-orbital modulation, and thus the precession period of the disc, it is crucial to obtain future monitoring observations of M51 ULX7. If the precession period is well maintained it would be evident of a stable clock, and the low luminosity states would only vary as a random walk (Staubert et al. 1983). However, a long monitoring could reveal drift in the start epoch of the low states similar to systems like Her X-1.

\section{CONCLUSION}

We have investigated the applicability of standard theoretical models to the observed properties of M51 ULX-7. The spin-up rate and mass accretion rate derived from observations are evident of a NS with a magnetic field of $2-7 \times 10^{13} \mathrm{G}$ rotating near equilibrium. Analysis of Swift/XRT archival data have shown the existence of a super-orbital period of $\sim 39 \mathrm{~d}$. Changes in the X-ray flux and spectral shape within the super-orbital period are most probably associated with disc precession that causes occultation and partial obscuration of the NS. Thus, no change in the accretion rate onto the NS is required to account for the super-orbital variability and mass transfer between the binary seems to remain fairly constant 
in timescales of years. If the super-orbital modulation is related to the NS free precession then the surface magnetic field strength of the NS should be $\sim 3-4 \times 10^{13} \mathrm{G}$. The agreement of these two independent estimates provides a strong argument for the NS free precession being responsible for the precession of the accretion disc in ULXs hosting magnetized NSs.

\section{ACKNOWLEDGEMENTS}

The authors would like to thank the anonymous referee for the the constructive report that helped to improve the manuscript. GV would like to thank M. Petropoulou for providing useful comments on the draft. We acknowledge the use of public data from the Swift data archive.

\section{REFERENCES}

Abolmasov P., Karpov S., Kotani T., 2009, PASJ, 61, 213

Andersson N., Glampedakis K., Haskell B., Watts A. L., 2005, MNRAS, 361,1153

Bachetti M., et al., 2014, Nature, 514, 202

Bachetti M., et al., 2019, arXiv e-prints, p. arXiv:1905.06423

Bardeen J. M., Petterson J. A., 1975, ApJ, 195, L65

Becker P. A., Wolff M. T., 2007, ApJ, 654, 435

Bonnet-Bidaud J. M., van der Klis M., 1981, A\&A, 97, 134

Burrows D. N., et al., 2005, Space Sci. Rev., 120, 165

Campana S., Stella L., Mereghetti S., de Martino D., 2018, A\&A, 610, A46

Carpano S., Haberl F., Maitra C., Vasilopoulos G., 2018, MNRAS, 476, L45

Chandrasekhar S., Fermi E., 1953, ApJ, 118, 116

Chashkina A., Abolmasov P., Poutanen J., 2017, MNRAS, 470, 2799

Chashkina A., Lipunova G., Abolmasov P., Poutanen J., 2019, A\&A, 626, A18

Christodoulou D. M., Laycock S. G. T., Yang J., Fingerman S., 2016, ApJ, 829,30

Clarkson W. I., Charles P. A., Coe M. J., Laycock S., Tout M. D., Wilson C. A., 2003, MNRAS, 339, 447

Dage K. C., Clarkson W. I., Charles P. A., Laycock S. G. T., Shih I. C., 2019, MNRAS, 482, 337

Dauser T., Middleton M., Wilms J., 2017, MNRAS, 466, 2236

Evans P. A., et al., 2007, A\&A, 469, 379

Evans P. A., et al., 2009, MNRAS, 397, 1177

Fabrika S., Ueda Y., Vinokurov A., Sholukhova O., Shidatsu M., 2015, Nature Physics, 11, 551

Foulkes S. B., Haswell C. A., Murray J. R., 2010, MNRAS, 401, 1275

Fürst F., et al., 2016, ApJ, 831, L14

Gehrels N., et al., 2004, ApJ, 611, 1005

Ghosh P., Lamb F. K., 1979a, ApJ, 232, 259

Ghosh P., Lamb F. K., 1979b, ApJ, 234, 296

Ghosh P., Lamb F. K., Pethick C. J., 1977, ApJ, 217, 578

Haberl F., Turolla R., de Vries C. P., Zane S., Vink J., Méndez M., Verbunt F., 2006, A\&A, 451, L17

Hickox R. C., Vrtilek S. D., 2005, ApJ, 633, 1064

Hickox R. C., Narayan R., Kallman T. R., 2004, ApJ, 614, 881

Ho W. C. G., Klus H., Coe M. J., Andersson N., 2014, MNRAS, 437, 3664

Horne J. H., Baliunas S. L., 1986, ApJ, 302, 757

Hu C.-P., Li K. L., Kong A. K. H., Ng C. Y., Lin L. C.-C., 2017, The Astrophysical Journal, 835, L9

Hung L.-W., Hickox R. C., Boroson B. S., Vrtilek S. D., 2010, ApJ, 720, 1202

Illarionov A. F., Sunyaev R. A., 1975, A\&A, 39, 185

Israel G. L., et al., 2017, Science, 355, 817

Jones D. I., 2012, MNRAS, 420, 2325

Jones D. I., Andersson N., 2001, MNRAS, 324, 811

Katz J. I., 1973, Nature Physical Science, 246, 87

King A., Lasota J.-P., 2019, MNRAS, 485, 3588
King A., Lasota J.-P., Kluźniak W., 2017, MNRAS, 468, L59

Koliopanos F., Vasilopoulos G., 2018, A\&A, 614, A23

Koliopanos F., Vasilopoulos G., Godet O., Bachetti M., Webb N. A., Barret D., 2017, A\&A, 608, A47

Kong A. K. H., Hu C.-P., Lin L. C.-C., Li K. L., Jin R., Liu C. Y., Yen D. C.-C., 2016, MNRAS, 461, 4395

Kosec P., Pinto C., Fabian A. C., Walton D. J., 2018a, MNRAS, 473, 5680

Kosec P., Pinto C., Walton D. J., Fabian A. C., Bachetti M., Brightman M., Fürst F., Grefenstette B. W., 2018b, MNRAS, 479, 3978

Lai D., 1999, ApJ, 524, 1030

Lai D., 2014, in European Physical Journal Web of Conferences. p. 01001 (arXiv: 1402 . 1903), doi:10.1051/epjconf/20136401001

Lander S. K., 2013, Phys. Rev. Lett., 110, 071101

Lander S. K., 2014, MNRAS, 437, 424

Lander S. K., Jones D. I., 2009, MNRAS, 395, 2162

Lau R. M., et al., 2019, ApJ, 878, 71

Lipunov V. M., Shakura N. I., 1980, Soviet Astronomy Letters, 6, 14

McQuinn K. B. W., Skillman E. D., Dolphin A. E., Berg D., Kennicutt R., 2016, ApJ, 826, 21

Middleton M. J., Heil L., Pintore F., Walton D. J., Roberts T. P., 2015, MNRAS, 447, 3243

Middleton M. J., et al., 2018a, arXiv e-prints, p. arXiv:1810.10518

Middleton M. J., et al., 2018b, MNRAS, 475, 154

Middleton M. J., Fragile P. C., Ingram A., Roberts T. P., 2019, MNRAS, 489, 282

Miller-Jones J. C. A., et al., 2019, Nature, 569, 374

Mönkkönen J., Tsygankov S. S., Mushtukov A. A., Doroshenko V., Suleimanov V. F., Poutanen J., 2019, A\&A, 626, A106

Mushtukov A. A., Suleimanov V. F., Tsygankov S. S., Poutanen J., 2015, MNRAS, 454, 2539

Mushtukov A. A., Suleimanov V. F., Tsygankov S. S., Ingram A., 2017, MNRAS, 467, 1202

Mushtukov A. A., Ingram A., Middleton M., Nagirner D. I., van der Klis M., 2019, MNRAS, 484, 687

Naik S., Paul B., 2003, Astronomy and Astrophysics, 401, 265

Ogilvie G. I., Dubus G., 2001, MNRAS, 320, 485

Parfrey K., Spitkovsky A., Beloborodov A. M., 2016, ApJ, 822, 33

Park T., Kashyap V. L., Siemiginowska A., van Dyk D. A., Zezas A., Heinke C., Wargelin B. J., 2006, ApJ, 652, 610

Paul B., Kitamoto S., Makino F., 2000, The Astrophysical Journal, 528, 410

Petterson J. A., 1975, ApJ, 201, L61

Pinto C., Middleton M. J., Fabian A. C., 2016, Nature, 533, 64

Pinto C., et al., 2017, MNRAS, 468, 2865

Postnov K., Shakura N., Staubert R., Kochetkova A., Klochkov D., Wilms J., 2013, MNRAS, 435, 1147

Poutanen J., Lipunova G., Fabrika S., Butkevich A. G., Abolmasov P., 2007, MNRAS, 377, 1187

Pringle J. E., 1996, MNRAS, 281, 357

Rappaport S. A., Fregeau J. M., Spruit H., 2004, ApJ, 606, 436

Rodríguez Castillo G. A., et al., 2019, arXiv e-prints, p. arXiv:1906.04791

Sathyaprakash R., et al., 2019, MNRAS, p. L104

Scargle J. D., 1982, ApJ, 263, 835

Shakura N. I., Sunyaev R. A., 1973, A\&A, 24, 337

Shakura N. I., Postnov K. A., Prokhorov M. E., 1998, A\&A, 331, L37

Shakura N. I., Prokhorov M. E., Postnov K. A., Ketsaris N. A., 1999, A\&A, 348,917

Stairs I. H., Lyne A. G., Shemar S. L., 2000, Nature, 406, 484

Stairs I. H., et al., 2019, MNRAS, 485, 3230

Staubert R., Bezler M., Kendziorra E., 1983, A\&A, 117, 215

Staubert R., Klochkov D., Postnov K., Shakura N., Wilms J., Rothschild R. E., 2009, A\&A, 494, 1025

Steiner A. W., Gandolfi S., Fattoyev F. J., Newton W. G., 2015, Phys. Rev. C, 91, 015804

Tananbaum H., Gursky H., Kellogg E. M., Levinson R., Schreier E., Giacconi R., 1972, ApJ, 174, L143

Truemper J., Kahabka P., Oegelman H., Pietsch W., Voges W., 1986, ApJ, 300, L63 
Tsygankov S. S., Mushtukov A. A., Suleimanov V. F., Poutanen J., 2016a, MNRAS, 457, 1101

Tsygankov S. S., Lutovinov A. A., Doroshenko V., Mushtukov A. A., Suleimanov V., Poutanen J., 2016b, A\&A, 593, A16

VanderPlas J. T., 2018, ApJS, 236, 16

Vasilopoulos G., Haberl F., Carpano S., Maitra C., 2018, A\&A, 620, L12

Vasilopoulos G., Petropoulou M., Koliopanos F., Ray P. S., Bailyn C. B., Haberl F., Gendreau K., 2019, MNRAS, 488, 5225

Walton D. J., et al., 2016, The Astrophysical Journal, 827, L13

Walton D. J., et al., 2018a, ApJ, 856, 128

Walton D. J., et al., 2018b, ApJ, 857, L3

Wang Y.-M., 1995, ApJ, 449, L153

West B. F., Wolfram K. D., Becker P. A., 2017, ApJ, 835, 129

Wijers R. A. M. J., Pringle J. E., 1999, MNRAS, 308, 207

Zhou Y., Feng H., Ho L. C., Yao Y., 2019, ApJ, 871, 115

This paper has been typeset from a $\mathrm{T}_{\mathrm{E}} \mathrm{X} / \mathrm{L} \mathrm{T}_{\mathrm{E}} \mathrm{X}$ file prepared by the author. 